\title{
¿Es concordante el tamaño tumoral medido en resonancia magnética mamaria y el de la biopsia quirúrgica en pacientes con cáncer de mama, tratadas con quimioterapia neoadyuvante?
}

Drs. Marcela Uchida $S^{(1)}$, Daniel Grudsky $\mathrm{P}^{(2)}$, Felipe Vergara $\mathrm{D}^{(2)}$, Miguel A. Pinochet $\mathrm{T}^{(1)}$, Eleonora Horvath ${ }^{(1)}$, Heriberto Wenzel $\mathrm{K}^{(1)}$, Eduardo Soto $\mathrm{N}^{(1)}$.

1. Servicio de Imágenes Mamarias, Departamento de Imágenes. Facultad de Medicina Clínica Alemana, Universidad del Desarrollo.

2. Facultad de Medicina Universidad del Desarrollo. Santiago - Chile.

Is breast MRI concordant with pathologic tumor size in breast cancer patients treated with neoadjuvant chemotherapy?

Abstract. Neoadjuvant chemotherapy (NACT) is a treatment used in those breast cancers initially inoperable due to their size, and also in operable breast cancers where NACT could increase the rate of conservative breast surgery. To assess tumor response to treatment, clinical examination, mammography, ultrasound, and breast MRI are used, the latter being the modality that yields the best correlation with histologic tumor volume. We evaluated the correlation of tumor sizes as measured by MRI versus surgical pathological specimen in breast cancers treated with NACT. Eighteen patients underwent MRI to monitor NACT; in 15 $(83 \%)$ of them the final biopsy was obtained. In this group a very good correlation was observed, with a mean difference between MRI and histology of $4 \mathrm{~mm}$ regarding tumor volumen, which has allowed an adequate management of patients in our daily practice.

Keywords: Breast MRI, Conservative breast surgery, Neoadjuvant chemotherapy.

Resumen. La quimioterapia neoadyuvante (QTNA) es un tratamiento usado en aquellos cánceres mamarios cuyo tamaño los hace inoperables al momento del diagnóstico y en cánceres mamarios operables, pero cuyo uso podría permitir una cirugía conservadora. Para evaluar la respuesta al tratamiento, se ha utilizado el examen clínico, la mamografía, el ultrasonido y la resonancia magnética mamaria, siendo ésta última la que mejor correlación tiene con el tamaño tumoral histológico. Quisimos evaluar la concordancia del tamaño tumoral medido en resonancia magnética con el de la biopsia quirúrgica, en cánceres mamarios tratados con QTNA. Dieciocho pacientes se realizaron resonancia magnética para monitorización de QTNA, en 15(83\%) de ellas se obtuvo la biopsia definitiva. En este grupo observamos una muy buena correlación, con una diferencia promedio de $4 \mathrm{~mm}$, entre el tamaño de la resonancia y el de la histología, lo que permitió en nuestro medio un adecuado manejo de las pacientes.

Palabras clave: Cirugía conservadora de la mama, Quimioterapia neoadyuvante, Resonancia magnética mamaria.

Uchida $\mathrm{M}$, et al. ¿Es concordante el tamaño tumoral medido en resonancia magnética mamaria y el de la biopsia quirúrgica en pacientes con cáncer de mama, tratadas con quimioterapia neoadyuvante? Rev Chil Radiol 2012; 18(2): 74-78.

Correspondencia: Dr. Daniel Grudsky / pdgrudsky@gmail.com

Trabajo recibido el 03 de abril de 2012, aceptado para publicación 01 de junio de 2012.

Introducción

La quimioterapia neoadyuvante (QTNA) es un tratamiento que se utiliza en los cánceres mamarios cuyo tamaño los hace inoperables al momento del diagnóstico y en cánceres mamarios operables, pero que al disminuir el tamaño tumoral se podría realizar una cirugía conservadora ${ }^{(1,2)}$.

Para evaluar la respuesta al tratamiento, se 
ha utilizado el examen clínico, la mamografía, el ultrasonido y la resonancia magnética mamaria.

La palpación es un método inexacto, ya que no diferencia lesión tumoral de estructuras adyacentes como la piel y los tejidos blandos y no discrimina entre fibrosis y tumor activo. La mamografía tampoco distingue tumor activo de fibrosis y si la densidad mamaria es alta, lo más probable es que no se distingan adecuadamente los márgenes tumorales. En cuanto al ultrasonido, éste no logra diferenciar tumor viable de fibrosis y además es un examen equipo y operador dependiente, lo que le otorga desventajas adicionales. La resonancia magnética en cambio, al utilizar medio de contraste, posibilita analizar tanto la morfología de la lesión, como la cinética de captación del contraste y por ende tener una mejor apreciación del tumor activo residual.

Es así como el coeficiente de correlación intraclase entre el tamaño tumoral supuesto y el histológico es de sólo 0.65 para el examen clínico, 0,69 para la mamografía 0,78 para el ultrasonido y llega a 0,97 para la resonancia magnética ${ }^{(3)}$.

A pesar de lo anterior también se ha visto que la resonancia magnética no es un método perfecto, describiéndose en las distintas series tanto subestimación (2-23\%), como sobrestimación (6-26\%) del tamaño tumoral real(4,5).

La importancia de una adecuada estimación del tamaño tumoral residual, radica en que permite obtener bordes quirúrgicos negativos, disminuye la morbilidad y optimiza el tipo de cirugía para la paciente.

Es importante destacar que en nuestro centro la indicación de monitorización de respuesta a la QTNA es muy baja, alcanzando menos de del $1 \%$ de la indicaciones de todas las resonancia mamarias.

\section{Objetivo}

Evaluar la concordancia del tamaño tumoral medido en resonancia magnética con el de la biopsia quirúrgica, en cánceres mamarios tratados con QTNA.

\section{Material y método}

Se seleccionaron aquellos casos de pacientes tratadas con QTNA, que se realizaron resonancia magnética en un periodo de 5 años. Se utilizó la base de datos Filemaker Pro 8,5 evaluándose datos demográficos, histología de la lesión, tamaño tumoral (diámetro mayor) y número de ciclos de quimioterapia. Todos los exámenes fueron realizados en resonador de 1,5 T (General Electric Medical Systems, Milwaukee, WI), con bobina de mama de 8 canales (Breast Array Coil for General Electric Signa System; MRI Devices, Waukesha, WI). Las secuencias utilizadas fueron: serie de T2 FAT/SAT derecha e izquierda, secuencia dinámica en T1: basal (máscara) y postcontraste en sagital
VIBRANT (Volume Imaging for Breast Assesment), adquisiciones de 1 a $7 \mathrm{~min}$, anulación de la grasa, matriz 256x256, FOV: $20 \mathrm{~cm}$, TR: $4 \mathrm{~ms}$, TE: $1.6 / \mathrm{fr}$ $\mathrm{ms}$, espesor de $3 \mathrm{~mm} /-1.5 \mathrm{~mm}$ (ZIP 2), contraste en dosis de $0,1 \mathrm{mmol}$ o $0,2 \mathrm{ml} / \mathrm{Kg}$ de Gd-DTPA, a un flujo de $2 \mathrm{ml} / \mathrm{seg}$, con bomba de infusión automática.

Todas las pacientes firmaron consentimiento informado antes de realizarse la resonancia magnética mamaria.

Las resonancias fueron informadas por alguno de los 7 radiólogos del servicio, en los informes se usaron los criterios RECIST (Response Evaluation Criteria In Solid Tumors) y se obtuvo el diámetro mayor de la lesión, en la resonancia más cercana a la cirugía, en general después del cuarto ciclo.

El análisis histológico fue hecho por un mismo patólogo de la institución, las muestras se procesaron en forma estándar y se seleccionó el diámetro histológico mayor.

Para el análisis estadístico se uso el método de Bland Altman y análisis comparativo mediante el coeficiente de correlación intraclase, considerándose una buena correlación si es mayor a 0.71 y significativo si la prueba se asocia a un $p$ menor a 0.05 .

\section{Resultados}

Dieciocho pacientes se realizaron resonancia magnética para monitorización de QTNA, en $15(83 \%)$ de ellas se obtuvo la biopsia definitiva y constituyen el grupo de análisis. La edad promedio fue 49,3 años (31 a 67), la mayoría $88,9 \%$ no tenía antecedentes familiares de cáncer de mama.

El $100 \%$ fueron cánceres ductales infiltrantes (CDI), 40\% fueron grado histológico II (Gráfico 1) y sólo un $6,7 \%$ fueron receptores hormonales triple negativos.

Las pacientes se sometieron en promedio a 4,2 (rango de 1 a 8) ciclos de QTNA.

El tamaño tumoral por resonancia promedio fue $32,33 \mathrm{~mm}$ (rango de 0 y $80 \mathrm{~mm}$ ) y el histológico de $36,93 \mathrm{~mm}$ (rango de 0 y $80 \mathrm{~mm}$ ) (Tabla I).

En dos casos se informó respuesta completa en resonancia, la histología lo confirmó en uno y en el otro se encontró un foco de $1 \mathrm{~mm}$. de carcinoma ductal in situ.

Al analizar el gráfico de Bland Altman se observa que hay muy buena concordancia entre el tamaño histológico y el de resonancia, con una diferencia promedio de $4 \mathrm{~mm}$ y si no tomamos en cuenta el caso en que la diferencia fue $38 \mathrm{~mm}$ entre el tamaño histológico y el de resonancia, la diferencia se reduce a solo $2 \mathrm{~mm}$.

La correlación intraclase arrojó un rho $=0.826$ con un intervalo de confianza $95 \%$ de 0.51-0.94, asociado a un $\mathrm{p}=0.001$. 
Tabla I.

\begin{tabular}{lccccc|}
$\begin{array}{l}\text { Diámetro } \\
\text { tumoral }\end{array}$ & $\begin{array}{c}\text { Número } \\
\text { pacientes }\end{array}$ & $\begin{array}{c}\text { Promedio } \\
(\mathrm{mm})\end{array}$ & $\begin{array}{c}\text { Desviación } \\
\text { estándar }\end{array}$ & Mínimo & Máximo \\
\hline Resonancia magnética & 15 & 32,33 & 22,96 & 0 & 80 \\
Histológico & 15 & 36,93 & 28,63 & 0 & 80
\end{tabular}

Distribución de resultado histología definitiva

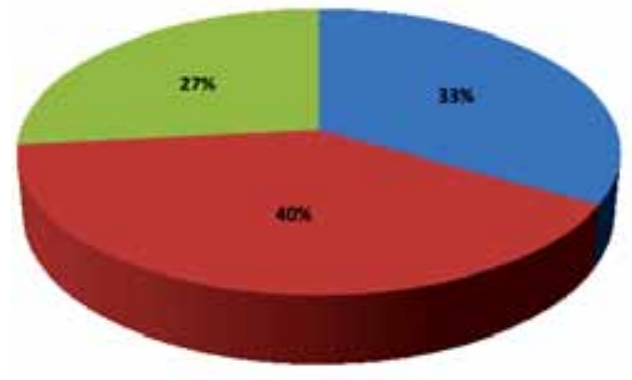

Gráfico de Bland-Altman (Representación diferencia promedios entre tamaño RM e histológico)

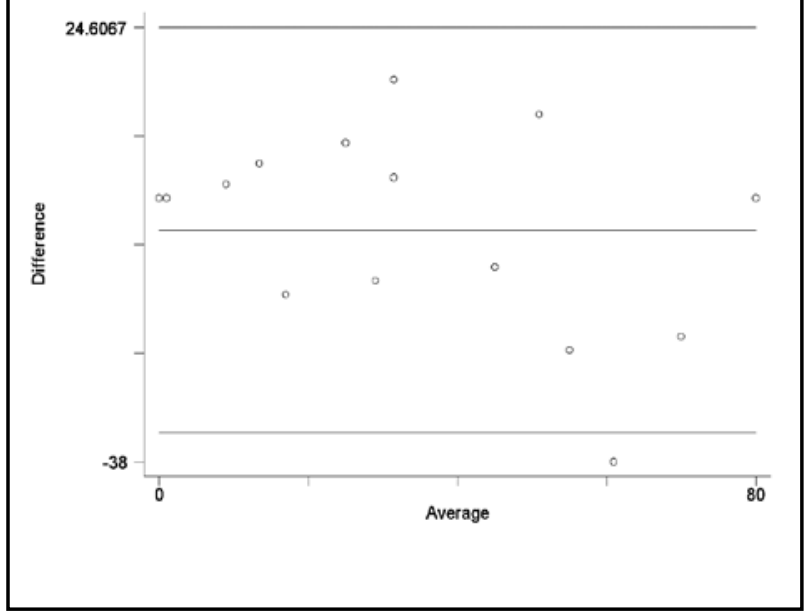

Limits of agreement (Reference Range for difference): -33.807 to 24.607 .

Mean difference: -4.600 ( $\mathrm{Cl}-12.687$ to 3.487$)$

Range: 0.000 to 80.000
Mostramos un ejemplo de un caso de una paciente de 31 años, con tumor palpable al momento del diagnóstico, cuya biopsia Core correspondió a un CDI GIII, triple negativo. El tamaño tumoral pretratamiento fue de $28 \mathrm{~mm}$. Después de cuatro ciclos de QTNA, se observa respuesta parcial, alcanzando el tamaño tumoral $12 \mathrm{~mm}$ y en la pieza operatoria de la misma paciente se observa un tamaño tumoral de $11 \mathrm{~mm}$ (Figuras 1, 2 y 3).

\section{Discusión}

La evaluación de enfermedad residual posterior a QTNA ayuda a determinar el pronóstico y planear el mejor tratamiento quirúrgico(6).

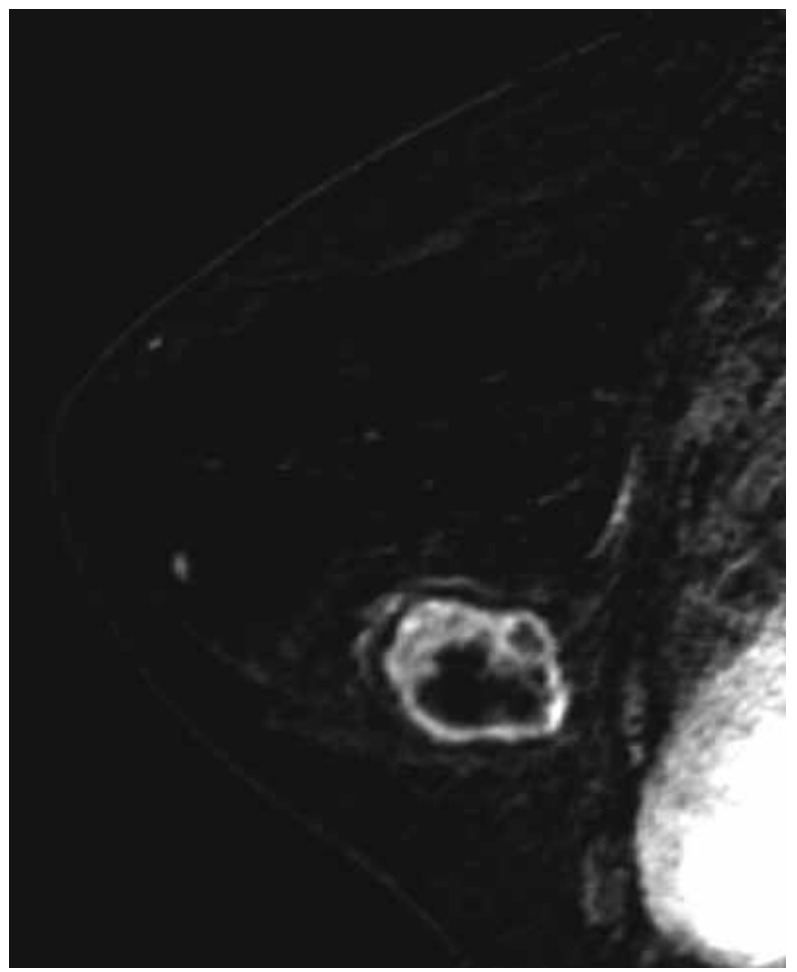

Figura 1. Resonancia magnética de paciente de 31 años con CDI GIII, triple negativo. Tamaño tumoral pre-tratamiento $28 \mathrm{~mm}$. 


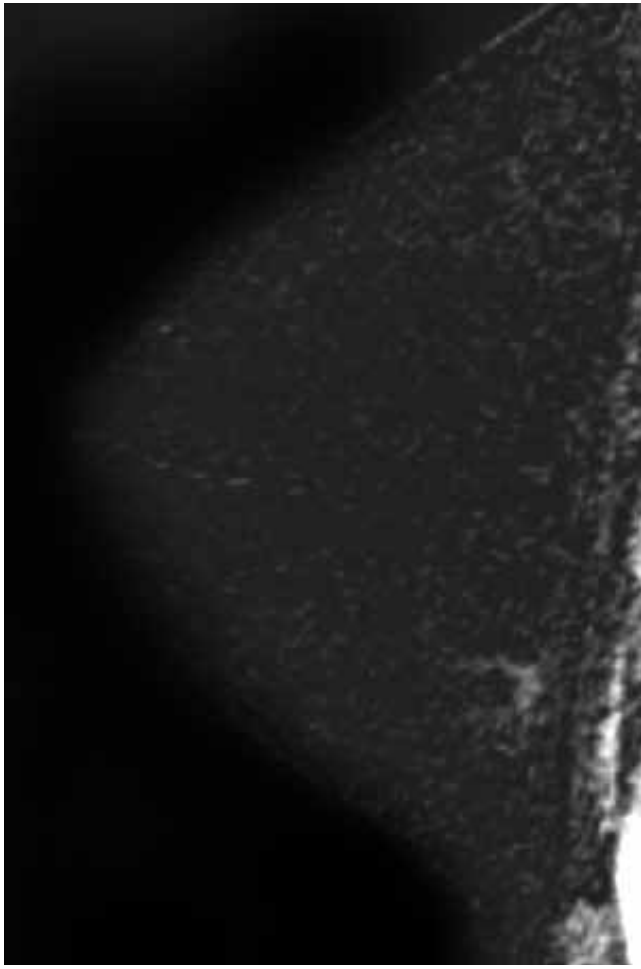

Figura 2. Resonancia magnética de la misma paciente al 4to ciclo de QTNA, tamaño tumoral post-tratamiento $12 \mathrm{~mm}$.

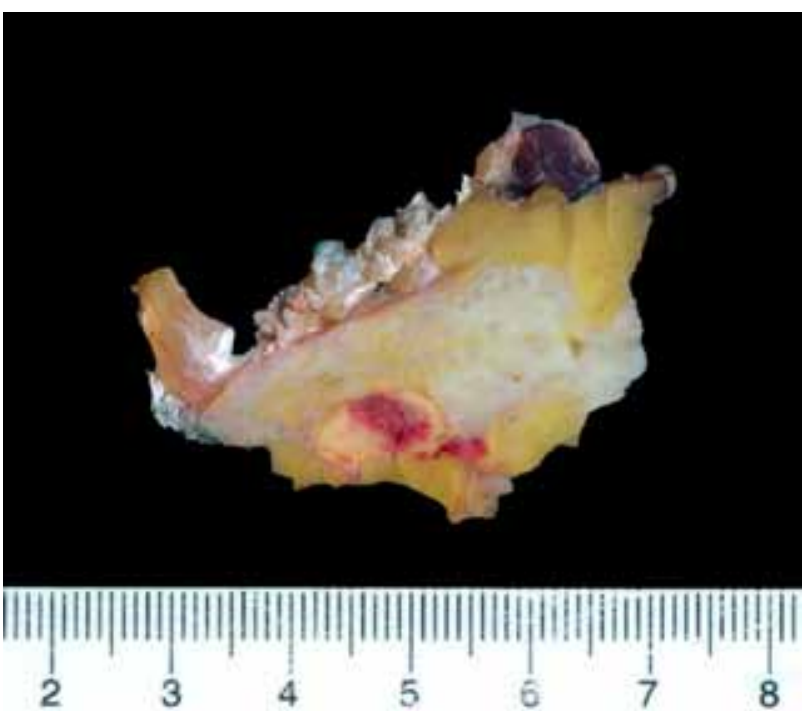

Figura 3. Fotografía de pieza operatoria, donde el tamaño tumoral es de $11 \mathrm{~mm}$.

Se ha visto que la resonancia magnética es un método confiable por su capacidad de analizar la morfología y cinética de la captación del contraste del tumor y en distintas series es más exacta que el examen clínico, la mamografía y el ultrasonido ${ }^{(3,7,8)}$.

En este trabajo se observó una excelente concordancia entre los tamaños medidos por resonancia magnética y el estándar de oro, que es el tamaño histológico, siendo la diferencia promedio de $4 \mathrm{~mm}$. Al excluir el único caso que se escapa del promedio y en el que la subestimación fue de $38 \mathrm{~mm}$, la diferencia se reduce sólo a $2 \mathrm{~mm}$, dentro de las explicacionespara esta subestimación, podría plantearse que fue una resonancia que se realizó cuando estábamos comenzando con la técnica en nuestro centro y donde además se registró artefacto de movimiento.

La fortaleza en disponer de una buena concordancia con el análsis histopatológico, permite al cirujano realizar un procedimiento quirúrgico en el que tendrá márgenes adecuados, con la disminución en la tasa de reoperaciones. El determinar márgenes libres de enfermedad es una gran ventaja de la resonancia magnética de evaluación de respuesta a QTNA, y su rol en esta entidad se refuerza con nuestros resultados.

Una limitante de nuestro estudio es que es una serie de casos pequeña para generalizar resultados.

\section{Conclusiones}

En nuestro medio observamos adecuada estimación del tamaño tumoral residual con resonancia magnética, mejorando el manejo de las pacientes.

Como desafíos a futuro tenemos que aumentar el número de casos y el seguimiento, además de analizar los cambios morfológicos, cinéticos de las lesiones y evaluar la utilidad de secuencias como la espectroscopía y difusión

\section{Bibliografía}

1. Mieog JS, van der Hage JA, van de Velde CJ. Neoadjuvant chemotherapy for operable breast cancer. Br J Surg 2007; 94: 1189-1200.

2. Rastogi P, Anderson SJ, Bear HD, et al. Preoperative chemotherapy: updates of National Surgical Adjuvant Breast and Bowel Project Protocols B-18 and B-27. J Clin Oncol 2008; 26: 778-785.

3. Shin HJ, Kim HH, Ahn JH, Kim SB, Jung KH, Gong $G$, et al. Comparison of mammography, sonography, MRI and clinical examination in patients with locally advanced or inflammatory breast cancer who underwent neoadjuvant chemotherapy. Br J Radiol. 2011; 84(1003): 612-620.

4. Vénat-Bouvet $L$, Desfougères $M$, Aubard $Y$, Mollard $J$, Fermeaux V, Genet D, et al. MRI evaluation of primary chemotherapy response in breast cancer. Bull Cancer 2004; 91: 721-728.

5. Kim HJ, Im YH, Han BK, Choi N, Lee J, Kim JH, et al. Accuracy of MRI for estimating residual tumor size after neoadjuvant chemotherapy in locally advanced breast cancer: relation to response patterns on MRI. Acta Oncol 2007; 46: 996-1000.

6. Kong X, Moran MS, Zhang N, Haffty B, Yang Q. 
Meta-analysis confirms achieving pathological complete response after neoadjuvant chemotherapy predicts favourable prognosis for breast cancer patients. Eur J Cancer. 2011; 47(14): 2084-2090.

7. Croshaw R, Shapiro-Wright H, Svensson E, Erb $\mathrm{K}$, Julian T. Accuracy of clinical examination, digital mammogram, ultrasound, and MRI in determining postneoadjuvant pathologic tumor response in operable breast cancer patients. Ann Surg Oncol. 2011; 18(11): 3160-3163. Epub 2011 Sep 27.

8. Keune JD, Jeffe DB, Schootman M, Hoffman A, Gillanders WE, Aft RL. Accuracy of ultrasonography and mammography in predicting pathologic response after neoadjuvant chemotherapy for breast cancer. Am J Surg. 2010; 199(4): 477484.

\section{CHILE EN LA CÚSPIDE DE LAS TECNOLOGÍAS DE RESONANCIA MAGNÉTICA}

Philips se enorgullece de presentar la primera y única tecnología 100\% digital en resonancia magnética: INGENIA.

Este 2012 es el año de arribo de esta evolución a Chile, lo que posiciona al sistema de salud de nuestro país a la vanguardia tecnológica mundial en resonancia magnética.

El uso de la resonancia magnética está creciendo rápidamente en los casos de rutina, y se suman a esto áreas emergentes como la oncología y la cardiología, mientras que los reembolsos se están reduciendo. INGENIA, el primer sistema digital de banda ancha es la respuesta de Philips a estos nuevos escenarios.

La compañía continuamente desarrolla tecnologías basadas en tres pilares fundamentales, que se resumen en la búsqueda de soluciones de integración y colaboración clínica; con foco completo en el paciente, haciendo inversiones sustentables y efectivas. Este concepto recibe el nombre de "Imaging 2.0". En este marco, INGENIA brinda múltiples elementos destacables alineados con los pilares antes mencionados: tecnología dStream 100\% digital que permite mejoras de calidad de imagen de hasta un $40 \%$ y una capacidad de expansión inmejorable; tecnología xTend que brinda el mayor confort al paciente; tecnología FlexStream que disminuyen los tiempos de estudio y mejoran el flujo de trabajo de los sistemas.

«Así como la Web 2.0 redefine la forma en que la gente interactúa, comparte y utiliza el Internet, el Imaging 2.0 representa un nuevo mundo de posibilidades para la ciencia de radiología. Se trata de la integración, colaboración y nuevos niveles de atención y seguridad del paciente que puede ayudar a los médicos a lograr lo que era inimaginable hace sólo unos pocos años"

INGENIA permite observar más cosas, con mayor detalle, y de forma más precoz; en un ambiente confortable y relajado para los pacientes; y comparte la información obtenida con otras modalidades de forma de lograr un mejor diagnóstico. Además tiene la capacidad permanecer a la vanguardia a lo largo del tiempo, lo que brinda a sus usuarios una solución práctica y rentable. Innovación, escalabilidad y actualización que permiten estar siempre vigentes

Algunos ejemplos de estas novedades las citamos en los siguientes elementos: primera utilización de antenas puramente digitales; considerable reducción de tiempo de preparación y de realización de estudios; características especiales de bobinas buscando maximizar la comodidad de los pacientes en los estudios, como la inclinación de la bobina de cabeza para pacientes que así lo requieran; ambientes de exámenes que buscan relajar a los pacientes a través de experiencias lumínicas, sonoras y visuales; y lo más importante: los métodos diagnósticos más vanguardistas para lograr la mayor sensibilidad y precisión diagnostica.

El equipo finalmente será inaugurado en Julio en las instalaciones del Hospital Clínico de la Pontificia Universidad Católica de Chile, iniciando así una nueva era en tecnología de resonancia magnética en nuestro país. 